\title{
Alemtuzumab Use in Clinical Practice: Recommendations from European Multiple Sclerosis Experts
}

\author{
Thomas Berger $^{1} \cdot$ Irina Elovaara $^{2} \cdot$ Sten Fredrikson $^{3} \cdot$ Chris McGuigan $^{4}$. \\ Lucia Moiola $^{5}$ - Kjell-Morten Myhr ${ }^{6}$ - Celia Oreja-Guevara ${ }^{7}$ Igor Stoliarov $^{8}$. \\ Uwe K. Zettl ${ }^{9}$
}

(C) The Author(s) 2017. This article is published with open access at Springerlink.com

\begin{abstract}
Alemtuzumab (Lemtrada ${ }^{\mathrm{TM}}$ ) is a humanized monoclonal antibody approved in more than 50 countries. Within the European Union, alemtuzumab is indicated for the treatment of adult patients with relapsing-remitting multiple sclerosis (RRMS) with active disease defined by clinical or imaging features; in the USA, the indication states that alemtuzumab should generally be reserved for the treatment of patients with relapsing forms of multiple sclerosis who have had an inadequate response to two or
\end{abstract}

Thomas Berger

thomas.berger@i-med.ac.at

1 Clinical Department of Neurology, Medical University of Innsbruck, Innsbruck, Austria

2 Department of Neurology and Rehabilitation, University of Tampere Medical School and Tampere University Hospital, Tampere, Finland

3 Department of Clinical Neuroscience, Karolinska Institute, Stockholm, Sweden

4 St Vincent's University Hospital, Dublin, Ireland

5 San Raffaele Scientific Institute, Milan, Italy

6 Haukeland University Hospital and University of Bergen, Bergen, Norway

7 Hospital Clínico San Carlos, Madrid, Spain

8 Institute of the Human Brain, Russian Academy of Sciences, St. Petersburg, Russia

9 Department of Neurology, Neuroimmunological Section, University of Rostock, Rostock, Germany more disease-modifying therapies (DMTs). In clinical trials, alemtuzumab demonstrated efficacy in treatment-naïve patients with active RRMS and those relapsing on prior DMTs, with a consistent and manageable safety and tolerability profile. The European Union indication provides physicians with significant flexibility regarding treatment decisions, affording the opportunity for individualized treatment. Thus, alemtuzumab may be an appropriate treatment choice across a broad range of patients with RRMS, including, for example, treatment-naïve patients with active disease, patients with highly active disease, or for patients relapsing on prior DMTs. There are several practicalities to consider when using alemtuzumab, including the unique dosing regimen, administered via intravenous infusion on 5 consecutive days at baseline and on 3 consecutive days 12 months later, and as-needed retreatment ( 3 consecutive days at least 12 months after the last course) in cases of disease recurrence. Additionally, routine monthly monitoring is required for up to 48 months after the last infusion to promptly identify potentially serious autoimmune adverse events. Given these considerations, it is beneficial to gain insight into how alemtuzumab is being used in the real-world clinical setting. Here, we report recommendations from European multiple sclerosis experts regarding best practices for alemtuzumab treatment, including management of adverse events and compliance with ongoing safety monitoring requirements. 


\section{Key Points}

In the European Union (EU), alemtuzumab is indicated for adult patients with relapsing-remitting multiple sclerosis, with active disease defined by clinical or imaging features. It can be considered as an initial therapeutic for treatment-naïve patients with active disease and for patients relapsing on prior disease-modifying therapy.

Healthcare providers should adhere to the alemtuzumab EU label, which gives a broad definition of patient eligibility for treatment; alemtuzumab is not suitable for patients with inactive relapsing-remitting multiple sclerosis, those stable on current therapy, or patients with progressive multiple sclerosis.

The Risk Management Program in the EU and other countries in addition to the Risk Evaluation and Mitigation Strategy in the USA are critical to ensure early detection of potential adverse events arising during and after alemtuzumab treatment and to ensure compliance with monitoring requirements. Data from an ongoing extension study, from realworld studies, and from post-marketing safety data will also be important to establish long-term safety of alemtuzumab treatment.

\section{Introduction}

Alemtuzumab is a humanized monoclonal antibody approved in more than 50 countries [1], including the European Union (EU) for the treatment of adult patients with active relapsing-remitting multiple sclerosis (RRMS) defined by clinical or imaging features, and the USA for the treatment of relapsing forms of multiple sclerosis (MS) $[2,3]$. In the USA, the indication for alemtuzumab states that it should generally be reserved for patients who have experienced an inadequate response to two or more disease-modifying therapies (DMTs) [3].

Alemtuzumab selectively targets CD52, a protein expressed at high levels on the surface of $\mathrm{T}$ and $\mathrm{B}$ lymphocytes but at lower levels on natural killer cells and other cell types involved in innate immunity, leading to a selective depletion of circulating $\mathrm{T}$ and $\mathrm{B}$ cells $[4,5]$, thereby decreasing inflammatory MS disease activity. Following treatment with alemtuzumab, T and B lymphocytes repopulate in a distinctive pattern over time that results in a rebalancing of the immune system over a period of 3-12 months [5, 6].

This mechanism allows for a unique dosing regimen of 12-mg intravenous infusions on 5 consecutive days at baseline and on 3 consecutive days 12 months later and may also account for the observed durable efficacy in the absence of continuous treatment, with most patients only requiring these two initial treatment courses $[2,7,8]$.

The safety and efficacy of alemtuzumab have been assessed in phase II and III clinical trials in treatment-naïve patients (CAMMS223 [NCT00050778]; CARE-MS I [NCT0053034 8]), in patients with active disease despite treatment with another DMT (CARE-MS II [NCT00548405]) (Table 1), and in extension (NCT00930553) and long-term follow-up studies (NCT02255656) that include patients from the phase II, III, and IV trials. The phase II and III studies all included an active comparator arm in which patients were treated with subcutaneous (SC) interferon $\beta$-1a (IFN $\beta$-1a), a DMT with an established efficacy across standard endpoints in RRMS [9-13]. In CAMMS223 and CARE-MS II, two doses (12 and $24 \mathrm{mg} /$ day) of alemtuzumab were evaluated; however, discussion of data in this article will be restricted to the 12-mg dose as this is the approved and commercially available dose $[2,9,12]$.

\section{Clinical Trial Experience with Alemtuzumab}

\subsection{Efficacy Data from Clinical Trials}

As discussed, in the three pivotal clinical trials, SC IFN $\beta$ 1a was included as an active comparator (Table 1); therefore, all subsequent comparisons relate to outcomes for alemtuzumab vs. SC IFN $\beta-1$ a. Across the CAMMS223 and CARE-MS I and II trials, alemtuzumab significantly reduced the annualized relapse rate (AAR) (co-primary endpoint) and was also associated with significant reductions in 6-month confirmed disability worsening (CDW, co-primary endpoint) in CAMMS223 [9] and CARE-MS II [12], and a non-significant $30 \%$ reduction in CARE-MS I [11].

In addition, in a recent analysis of data from the CAMMS223 study, alemtuzumab had greater efficacy than SC IFN $\beta-1 a$ at month 36 in each of the functional systems that make up the Expanded Disability Status Scale (EDSS) score, with the greatest effects being observed in the sensory, pyramidal, and cerebellar systems, which are thought to drive CDW in RRMS [16]. Across all trials, alemtuzumab also demonstrated improvements in several magnetic resonance imaging (MRI) outcomes (lesion volume/load, and brain atrophy) $[9,11,12]$.

In CARE-MS I and II, alemtuzumab-treated patients demonstrated a reduced rate of brain atrophy, as determined by median yearly percentage change in brain 
Table 1 Alemtuzumab clinical trial program

\begin{tabular}{|c|c|c|c|c|c|}
\hline \multicolumn{2}{|c|}{ 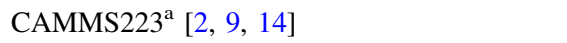 } & \multicolumn{2}{|c|}{ CARE-MS I $[2,11,14]$} & \multicolumn{2}{|c|}{ CARE-MS II $^{\mathrm{a}}[2,12,14]$} \\
\hline \multicolumn{4}{|c|}{ Patients with active RRMS who were treatment naïve } & \multirow{2}{*}{\multicolumn{2}{|c|}{$\begin{array}{l}\text { Patients with active RRMS who relapsed on } \\
\text { prior DMT } \\
\text { Study duration: } 2 \text { years }\end{array}$}} \\
\hline \multicolumn{2}{|c|}{ Study duration: 3 years } & \multicolumn{2}{|c|}{ Study duration: 2 years } & & \\
\hline $\begin{array}{l}\text { SC IFN } \beta-1 \mathrm{a} \\
(44 \mu \mathrm{g} \text { TIW }) \\
N=111\end{array}$ & $\begin{array}{l}\text { Alemtuzumab } \\
(12 \mathrm{mg} / \text { day }) \\
N=112\end{array}$ & $\begin{array}{l}\text { SC IFN } \beta-1 \mathrm{a} \\
(44 \mu \mathrm{g} \text { TIW }) \\
N=187\end{array}$ & $\begin{array}{l}\text { Alemtuzumab } \\
(12 \mathrm{mg} / \text { day }) \\
N=376\end{array}$ & $\begin{array}{l}\text { SC IFN } \beta-1 \mathrm{a} \\
(44 \mu \mathrm{g} \text { TIW }) \\
N=202\end{array}$ & $\begin{array}{l}\text { Alemtuzumab } \\
(12 \mathrm{mg} / \text { day }) \\
N=426\end{array}$ \\
\hline \multicolumn{2}{|c|}{$\begin{array}{l}\text { MRI criteria: diagnosis per McDonald } 2001 \\
\text { criteria, including brain MRI; } \geq 1 \mathrm{Gd}^{+} \text {lesion } \\
\text { on any of } \leq 4 \text { brain scans during } \leq 3 \text {-month } \\
\text { run-in period (including baseline scan) }\end{array}$} & \multicolumn{2}{|c|}{$\begin{array}{l}\text { MRI criteria: diagnosis per McDonald } 2005 \\
\text { criteria; brain MRI scan demonstrating white } \\
\text { matter lesions attributable to MS (within } 5 \\
\text { years of screening) }\end{array}$} & \multicolumn{2}{|c|}{$\begin{array}{l}\text { MRI criteria: diagnosis per McDonald } 2005 \\
\text { criteria; white matter lesions attributable to } \\
\text { MS and at least one of the following: } \geq 9 \mathrm{~T}_{2} \\
\text { lesions } \geq 3 \mathrm{~mm} \text {, any axis; } \mathrm{Gd}^{+} \text {lesion } \geq 3 \\
\text { mm, any axis, with } \geq 1 \text { brain } \mathrm{T}_{2} \text { lesion; } \\
\text { spinal cord lesion with } \geq 1 \text { brain } \mathrm{T}_{2} \text { lesion }\end{array}$} \\
\hline \multicolumn{2}{|c|}{$\begin{array}{l}\text { Active MS: } \geq 2 \text { relapses in the prior } 2 \text { years } \\
\text { and } \geq 1 \mathrm{Gd}^{+} \text {MRI lesion at screening }\end{array}$} & \multicolumn{2}{|c|}{$\begin{array}{l}\text { Active MS: } \geq 2 \text { relapses in the prior } 2 \text { years, } \\
\text { with } \geq 1 \text { relapse occurring in the year prior } \\
\text { to study entry }\end{array}$} & \multicolumn{2}{|c|}{$\begin{array}{l}\text { Active MS: } \geq 2 \text { relapses in the prior } 2 \text { years, } \\
\text { with } \geq 1 \text { relapse occurring in the year prior } \\
\text { to study entry and } \geq 1 \text { relapse occurring } \\
\text { during prior treatment } b\end{array}$} \\
\hline \multicolumn{2}{|c|}{ Mean age: 32 years } & \multicolumn{2}{|c|}{ Mean age: 33 years } & \multicolumn{2}{|c|}{ Mean age: 35 years } \\
\hline \multicolumn{2}{|c|}{ EDSS range: $0.0-3.0(\text { mean } 2.0)^{\mathrm{c}}$} & \multicolumn{2}{|c|}{ EDSS range: $0.0-3.0(\text { mean } 2.0)^{\mathrm{d}}$} & \multicolumn{2}{|c|}{ EDSS range: $0.0-5.0(\text { mean } 2.7)^{\mathrm{d}}$} \\
\hline \multicolumn{2}{|c|}{$\begin{array}{l}\text { Mean/median time since first MS episode: } 1.4 / \\
1.3 \text { years }\end{array}$} & \multicolumn{2}{|c|}{$\begin{array}{l}\text { Mean/median time since first MS episode: } 2.0 / \\
1.6 \text { years }\end{array}$} & \multicolumn{2}{|c|}{$\begin{array}{l}\text { Mean/median time since first MS episode: } 4.5 \text {, } \\
3.8 \text { years }\end{array}$} \\
\hline
\end{tabular}

Retreatment criteria for CAMMS223 (Sanofi Genzyme, data on file) and CARE-MS extensions [15]: $\geq 1$ relapse or $\geq 2$ new or enlarging $T_{2}$ and/ or $\mathrm{Gd}^{+}$brain or spinal lesions, $\geq 12$ months since the second alemtuzumab course

$D M T$ disease-modifying therapy, EDSS Expanded Disability Status Scale, $G d^{+}$gadolinium-enhancing, $I F N$ interferon, $M R I$ magnetic resonance imaging, $M S$ multiple sclerosis, $R R M S$ relapsing-remitting multiple sclerosis, $S C$ subcutaneous, $T I W$ three times per week

${ }^{a}$ A 24-mg/day treatment arm was included in these studies

b Treatment with IFN $\beta$ or glatiramer acetate for $\geq 6$ months

$c$ At screening and baseline visits

d At screening

parenchymal fraction over 2 years [11, 17], reaching significance in both CARE-MS I $(42 \%$ reduction, $p<0.0001)$ and CARE-MS II (24\% reduction, $p=0.0121)$ compared with SC IFN $\beta-1$ a at year 2. Furthermore, durable efficacy (AAR, disability, and MRI outcomes, including lesions and brain atrophy) was demonstrated throughout the extension studies [7, 8, 13, 17-19], with the majority of patients (68-94\%) not requiring retreatment with alemtuzumab or another DMT [17, 20]. Key efficacy outcomes from the clinical trial program trials are summarized in Table 2.

\subsection{Safety Data from Clinical Trials}

Alemtuzumab has a consistent and manageable safety and tolerability profile as demonstrated across individual clinical trials $[9,11,12])$, enrolling a total of 1694 patients. Furthermore, a similar safety and tolerability profile was also documented with a long-term follow-up (up to 5 years) in the extension studies $[7,8,13]$.

Nevertheless, several adverse events (AEs) of interest have been reported (Table 3 ). The most frequently reported AEs in clinical trials were infusion-associated reactions
(IARs), experienced by $>90 \%$ of patients, which peaked immediately following the initial alemtuzumab course and then decreased with subsequent courses [21, 22]. Few $(\leq 3 \%)$ serious IARs were reported [9, 11-13, 21, 23, 24].

The incidence of infections (which were mainly mild to moderate in severity) was greatest during the first month following infusion in all three trials [25] but was lower in the CAMMS223 and CARE-MS extensions compared with the core studies, suggesting a reduction in infection risk over time [11-13, 15].

Thyroid disease was the most common autoimmune event; however, $<1 \%$ of patients experienced serious thyroid AEs [2, 9, 13, 26, 27]. Immune thrombocytopenic purpura (ITP) was identified as a potential risk in the CAMMS223 study, initially reported in six patients, including the fatal index case in a patient receiving alemtuzumab $24 \mathrm{mg}[9,28,29]$. Across all clinical trials, ITP incidence was $2 \%$ in patients receiving alemtuzumab 12 or $24 \mathrm{mg}$ (1.6\% in patients receiving alemtuzumab $12 \mathrm{mg})$ [11-13, 29].

In response to the index case and other autoimmune events, enhanced monitoring and patient education was 


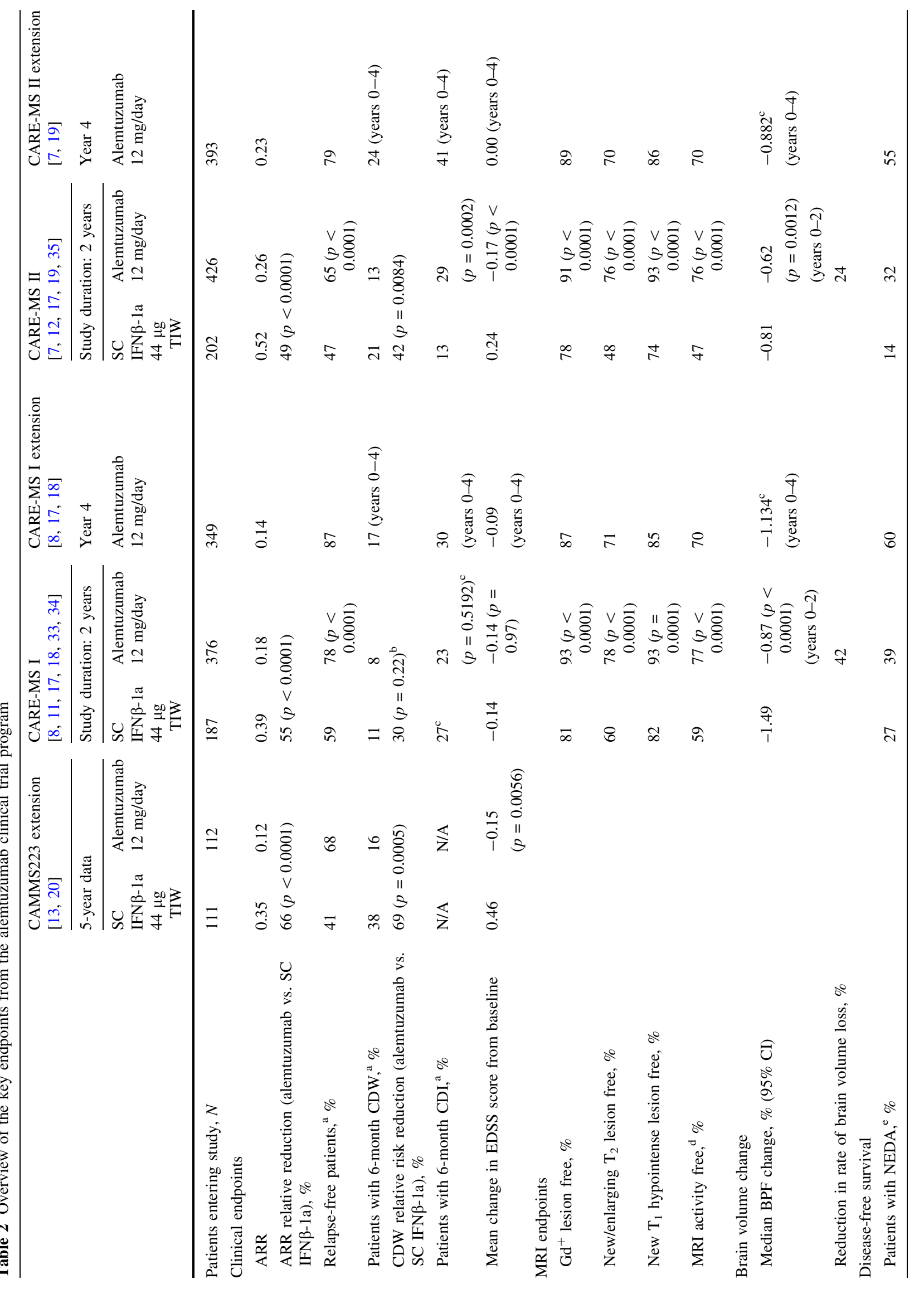




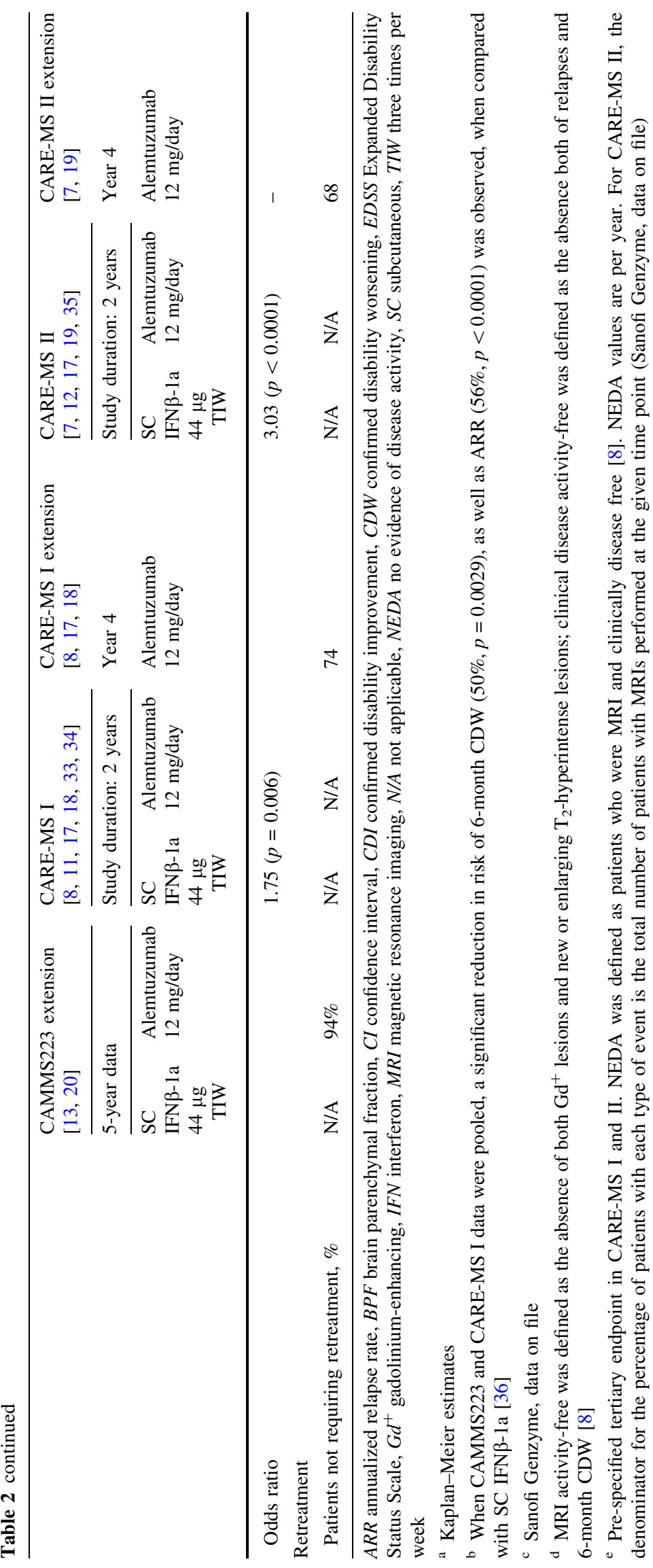


Table 3 Most common AEs observed in clinical trials of alemtuzumab $12 \mathrm{mg}$

\begin{tabular}{llll}
\hline & $\begin{array}{l}\text { CAMMS223 } \\
{[9,10]} \\
N=108\end{array}$ & $\begin{array}{l}\text { CARE-MS I } \\
{[11]} \\
N=376\end{array}$ & $\begin{array}{l}\text { CARE-MS II } \\
{[12]} \\
N=435\end{array}$ \\
\hline AEs occurring in $>10 \%$ of patients, $n(\%)$ & & & \\
$\quad$ Infusion-associated reactions & $106(98)$ & $338(90)$ & $393(90)$ \\
Infection & $71(66)$ & $253(67)$ & $334(77)$ \\
Upper respiratory tract & $48(44)$ & $57(15)$ & $71(16)$ \\
Urinary tract & $10(9)$ & $64(17)$ & $93(21)$ \\
Autoimmunity & & & \\
Any autoimmune thyroid-associated event & $28(26)$ & $68(18)$ & $69(16)$ \\
Blood and lymphatic system disorders & $\mathrm{NR}$ & $66(18)$ & $59(14)$ \\
Lymphopenia & $\mathrm{NR}$ & $26(7)$ & $23(5)$ \\
Leukopenia & $\mathrm{NR}$ & $11(3)$ & $\mathrm{NR}$ \\
\hline
\end{tabular}

$A E s$ adverse events, $N R$ not reported introduced for ongoing trials, which resulted in early identification and management of ITP cases, as well as other autoimmune events (thyroid dysfunction and glomerulonephropathy) in the clinical trials and improved patient outcomes. Subsequently, a Risk Management Program (RMP) and a Risk Evaluation and Mitigation Strategy (REMS) [30] were implemented to ensure early detection of potential AEs in real-world clinical practice. Across the clinical development program, there were four reported cases of immune nephropathy, including one case of antiglomerular basement membrane (anti-GBM) disease (in CAMMS223) reported 39 months after the second alemtuzumab course [13, 31].

A single fatal case of progressive multifocal leukoencephalopathy (PML) has been reported in a patient who switched from natalizumab to alemtuzumab. The PML diagnosis was not made until after the patient had received the first course of alemtuzumab; however, retrospective analysis of the MRI data showed that the onset of PML predated alemtuzumab treatment and, therefore, was attributed to natalizumab treatment (Sanofi Genzyme, data on file). The efficacy and safety outcomes from the alemtuzumab clinical trials have also been extensively reported in the literature; for further information, we refer the reader to these publications [9, 11-13, 32].

\subsection{Additional Long-Term Experience with Alemtuzumab}

A long-term, investigator-led, observational cohort study has also demonstrated the long-term efficacy and safety of alemtuzumab in patients with active RRMS [37]. In this study, 87 patients (39\% having received a prior DMT) were followed for up to 12 years (median follow-up 7 years). The majority of patients $(68 \%)$ experienced an improvement or stabilization of disability (based upon 6-month
CDW) compared with baseline. Retreatment was permitted in the event of a relapse with $52 \%$ of patients receiving only the initial two courses, with the remainder receiving three $(36 \%)$, four $(8 \%)$, or five $(1 \%)$ courses. The remaining patients $(3 \%)$ received only a single treatment course [37].

\section{Use of Alemtuzumab in a Real-World Clinical Setting}

Real-world data indicate that, in the majority of patients with active or highly active RRMS, alemtuzumab treatment is associated with disease stabilization [37, 38]. There is, however, some debate regarding which patients would benefit most from alemtuzumab treatment. The following sections of this article present the opinions and recommendations of European MS experts with regard to identifying the most appropriate patients for alemtuzumab treatment and best practices for treatment and monitoring.

\subsection{Alemtuzumab Indication}

The EU indication for alemtuzumab states that alemtuzumab is suitable for "adult patients with RRMS with active disease defined by clinical or imaging features" [2]. This represents an apparent shift from previous regulatory labeling on the eligibility of patients for a particular DMT and potentially allows physicians to use alemtuzumab as a first-line treatment choice in appropriate patients. EU indications for other DMTs (e.g., fingolimod, natalizumab) are more restrictive, requiring evidence of a specified level of MRI activity, in addition to clinical activity before treatment initiation. Fingolimod and natalizumab are therefore recommended for use in patients with highly active disease who have breakthrough disease activity on a 
Table 4 Summary of author experiences with alemtuzumab

\begin{tabular}{ll}
\hline National approval/reimbursement period & September 2013-May 2015 \\
Patients treated, $N$ & 181 \\
Female, $n(\%)$ & $129(71)$ \\
Age, mean (range), years & $35(17-66)$ \\
Alemtuzumab as: & \\
$\quad$ First-line therapy, $n(\%)$ & $27(15)$ \\
Escalation therapy, $n(\%)$ & $154(85)$ \\
\hline
\end{tabular}

Data provided courtesy of the authors and represents a summary of the experiences in Austria, Finland, Germany, Ireland, Italy, Norway, and Spain previous DMT (i.e., as a second-line therapy) or in those with more rapidly evolving severe RRMS [39, 40].

\subsection{Is There a Requirement for Specific Patient Profiles?}

The EU indication for alemtuzumab may provide prescribing physicians with certain challenges when deciding which patients are most suitable for treatment, although it also provides physicians with flexibility, allowing them to use their own clinical experience and judgment to make an informed decision regarding what constitutes active disease and to provide individualized treatment choices in collaboration with their patients. Clinical experience may be more valuable than rigid treatment guidelines, which attempt to define specific patient profiles for treatment eligibility. Furthermore, defining a single specific patient profile for alemtuzumab may not be helpful as this may unintentionally restrict the use of alemtuzumab in certain patients, particularly given that alemtuzumab efficacy has been demonstrated in a broad range of patients and that subgroup analyses have shown that the effects of alemtuzumab remain consistent across most demographic and disease characteristic subgroups [41-43].

Therefore, further studies are required to elucidate the variability and durability of response in patients treated with alemtuzumab to identify reliable biomarkers or clinical characteristics that can assist in the management of MS and further inform treatment decisions. Recently, several groups have investigated the prognostic value of peripheral $\mathrm{CD} 4^{+}$lymphocyte cell count recovery as a potential biomarker to identify patients who might benefit from retreatment with an additional course of alemtuzumab, although the evidence is conflicting in this regard [44-46].

\subsection{Personal Experience with Alemtuzumab in Routine Clinical Practice}

In the absence of specific patient profiles and validated biomarkers, personal clinical experience will inevitably influence alemtuzumab-related treatment decisions. Collectively, we have treated 181 patients with alemtuzumab, either as a first-line or as an escalation therapy in patients with breakthrough disease activity on a previous DMT (Table 4).

Most of our patients initiated treatment with alemtuzumab owing to breakthrough disease (i.e., having only a partial response to, or not responding to, other therapies). In general, these patients had experienced one or more relapses within the previous 12 months and demonstrated recent inflammatory disease activity (as evidenced either by gadolinium enhancement $\left(\mathrm{Gd}^{+}\right)$or by an obvious increase in $\mathrm{T}_{2}$ lesion load) on a brain MRI. Thus, alemtuzumab use in this group of patients was consistent with a treatment escalation paradigm. There was a consensus that more favorable treatment outcomes are typically observed in these patients if alemtuzumab treatment can be initiated early on in the disease course, particularly in patients who are younger, have highly active disease, and have low levels of disability at the start of treatment. Nevertheless, in clinical practice, we have also found alemtuzumab is efficacious in patients with already accumulating disability, particularly when used as a rescue therapy to stabilize disease and prevent further disability worsening if inflammatory disease activity (either clinically or on MRI) is still overt. We, therefore, recommend the use of alemtuzumab in patients with active RRMS, regardless of their level of disability.

However, $15 \%$ of our patients represented a very important treatment group, namely those who were treatment naïve but who presented with early, highly active disease. Compared with patients receiving alemtuzumab as an escalation therapy, these patients were generally younger and had a shorter, but more active disease course, usually with two or more relapses in the preceding 3-6 months (cluster of relapses). We feel that alemtuzumab may represent an effective treatment option in treatmentnaïve patients with rapidly evolving MS (or a clinical relapse accompanied by an increase in the number of $\mathrm{T}_{2}$ lesions and/or ongoing evidence of $\mathrm{Gd}^{+} \mathrm{T}_{1}$ lesions), and, as experience grows and the favorable outcomes associated with early intervention with alemtuzumab become evident, alemtuzumab use in this patient population will increase. In CARE-MS I, alemtuzumab significantly reduced the rate of 
brain volume loss in treatment-naïve patients with MS by $42 \%$ compared with SC IFN $\beta-1$ a [34], and, given the correlation between brain volume loss and disability and cognitive worsening [47, 48], early treatment with alemtuzumab may be more favorable than delaying treatment (discussed in Sect. 3.4). Indeed, we feel that initiating alemtuzumab in treatment-naïve patients may be advantageous, as lymphocyte levels have not been affected by use of prior DMTs.

In our experience, alemtuzumab has a place in routine clinical practice for the treatment of patients relapsing on prior treatments, as well as those who are treatment naïve. Postponing treatment in favor of escalating patients through alternative DMTs, in effect retaining alemtuzumab as a last resort, is not advised, and we feel that initiating alemtuzumab as soon as possible, particularly in patients with low levels of disability, will be associated with the most favorable outcomes.

\subsection{Alemtuzumab Early in Multiple Sclerosis}

The importance of treating MS early in the disease course to prevent inflammatory processes that lead to irreversible brain loss is well established [49-52]. Traditionally, the treatment paradigm is one of escalation therapy, during which drugs with greater efficacy (often with distinct mechanisms of action) but with increasing risk are used as disease progresses, with the most efficacious drugs (e.g., natalizumab, often considered following failure of one or more DMTs [53, 54]) used as the last line of therapy. However, data from alemtuzumab clinical studies [9, 11-13], coupled with its indication in the EU [2], afford physicians the opportunity to start alemtuzumab treatment in patients with active MS early in the disease course to prevent potentially avoidable CNS damage and provide the patient with the best opportunity for favorable treatment outcomes. This may be particularly important for patients with active MS with poor prognostic signs, for example, patients presenting with motor, cerebellar, or sphincter involvement at onset or those experiencing frequent relapses with poor recovery during the early years of their disease [55, 56]. The benefits of early intervention with other DMTs have been widely reported; for example, in the pivotal 2-year SC IFN $\beta$-1a study (PRISMS) and its 2-year extension (PRISMS-4), patients who started treatment early had improved clinical outcomes compared with patients whose treatment was delayed $[57,58]$. These observations have been confirmed in long-term follow-up studies [59-61]. Early intervention is thought to address the inflammatory component of the disease, thereby reducing development of further CNS pathology [53, 62]. Aggressive therapy early on in the disease course may provoke an immunological reset and may, therefore, favorably affect long-term disease progression [53].

However, the concept of early treatment of active disease with an immunomodulatory drug such as alemtuzumab, believed to rebalance the immune system, is not yet fully established and would represent a significant change in mindset for some physicians. In some European specialist MS centers, patients with highly active or rapidly evolving severe RRMS are already considered for first-line treatment with fingolimod or natalizumab (both considered typically second-line therapies in the EU), and, as noted above, alemtuzumab was used as a first-line therapy in $15 \%$ of all alemtuzumab-treated patients in our experience (Table 4). These observations perhaps indicate that MS treatment may be moving into a new era, away from the escalation paradigm and toward more robust early treatment of active disease.

\subsection{Switching to Alemtuzumab from Prior Disease- Modifying Therapies}

The efficacy of alemtuzumab in patients with active disease who had relapsed on prior DMTs is of particular clinical relevance; in such cases, switching therapies should be considered urgently to bring MS activity under control. As discussed briefly above (Sect. 2.1, Table 2), the CARE-MS II study demonstrated superior efficacy with alemtuzumab vs. SC IFN $\beta-1 \mathrm{a}$ in patients with active disease who had relapsed on prior DMTs [12]. The opportunity to switch therapies may be particularly important for certain patient subgroups. For example, patients receiving natalizumab therapy for over 2 years and/or who are positive for antiJohn Cunningham virus (anti-JCV) antibodies, as well as those having previously received other immunosuppressive medications, are at increased risk of developing PML and may require an alternative DMT [63]. Here, too, alemtuzumab may provide a treatment alternative option.

However, transitioning from one particular DMT to another can be complex, and a washout period may be required in certain circumstances. Treatment cessation guidelines and recommended washout periods are sometimes provided within the respective label of each DMT (Table 5), although there are currently no recommendations for transitioning to alemtuzumab from these individual DMTs. However, in the CARE-MS II extension, patients who switched from SC IFN $\beta$-1a to alemtuzumab were not required to undergo a washout period. Therefore, it is often unclear for which treatment transitions a washout period is required, how long it should be, or what long-term safety surveillance procedures should be implemented [53]. Nevertheless, as with other immunomodulatory therapies, concomitant treatment, including initiation of alemtuzumab within the washout period of the previous DMT, 
Table 5 General guidance for therapy cessation for common DMTs

\begin{tabular}{|c|c|}
\hline \multirow{2}{*}{$\begin{array}{l}\text { Interferons, glatiramer } \\
\text { acetate }\end{array}$} & No specific guidance for cessation of therapy ${ }^{a}$ \\
\hline & No washout period recommended based upon the mechanism of action [64] \\
\hline \multirow[t]{2}{*}{ Teriflunomide } & An AEP is available if rapid removal of teriflunomide from the circulation is desired [65] \\
\hline & $\begin{array}{l}\text { AEP will reduce plasma concentrations to } 0.02 \mathrm{mg} / \mathrm{L} \text { in } 11 \text { days. Complete elimination requires } 8 \text { months to } 2 \text { years in } \\
\text { the absence of AEP }[65,66]\end{array}$ \\
\hline Dimethyl fumarate & No specific guidance for cessation of therapy or requirement for washout [67] \\
\hline Fingolimod & A 6-week treatment-free period is required to clear fingolimod from circulation [39] \\
\hline Natalizumab & $\begin{array}{l}\text { A washout period might be appropriate as the pharmacodynamic effects of natalizumab last for approximately } 12 \\
\text { weeks following the last dose [40] }\end{array}$ \\
\hline Daclizumab & Washout period of 4 weeks is recommended ${ }^{\mathrm{b}}[68]$ \\
\hline Rituximab/ocrelizumab $^{c}$ & Washout period of 6 months is recommended ${ }^{\mathrm{b}}[68]$ \\
\hline
\end{tabular}

$A E P$ accelerated elimination procedure, $D M T s$ disease-modifying therapies, $E U$ European Union, $F A C S$ fluorescence-activated cell sorting, $I F N$ interferon, $M S$ multiple sclerosis, $S C$ subcutaneous

a In CARE-MS II [12], no washout period was required for patients switching from SC IFN $\beta-1$ a to alemtuzumab

b Washout guidance is based on cited sources along with the expert opinion of the authors

c Ocrelizumab is not yet approved for the treatment of MS in the EU

In all cases, the immune competence (including FACS analysis for B cells in the case of rituximab/ocrelizumab) should be confirmed before initiating alemtuzumab

is not advisable, owing to the potential risk of carry-over PML from previous treatment and additive effects on the immune system [2]. Consequently, it is important to consider the half-life as well as the mechanism of action (MoA) of the previous DMT when transitioning to alemtuzumab [53].

Despite the absence of specific guidance for switching to alemtuzumab from the DMTs listed in Table 5, there are some considerations that may help guide switching in clinical practice. The proposed MoA of fingolimod (preventing lymphocyte egress from peripheral lymphoid organs) results in low levels of circulating lymphocytes [69]. Therefore, it may be advisable to wait until lymphocyte counts begin to recover before initiating treatment with a DMT, such as alemtuzumab, particularly given that the proposed MoA of alemtuzumab in MS requires effective targeting of circulating $\mathrm{T}$ and $\mathrm{B}$ cells, leading to their depletion and subsequent repopulation [4, 5, 64]. Indeed, a recent report demonstrated clinical and MRI disease activity in alemtuzumab-treated patients who had switched from fingolimod but for whom a potentially insufficient washout period following fingolimod cessation had been used, resulting in lymphocyte counts below normal levels at the time of alemtuzumab treatment. The authors hypothesized that the sequestration of lymphocytes in lymph nodes, owing to the mechanism of action of fingolimod, coupled with an inadequate washout period (median 6 weeks, range 4-10 weeks) may have reduced the effectiveness of alemtuzumab [70].

By contrast, the proposed MoA of dimethyl fumarate (DMF) (activation of the nuclear factor erythroid 2-related factor 2) would generally not predict any issues with rapid transition to another therapy, although DMF has also been shown to have a lymphopenic effect in certain patient populations. Thus, as for fingolimod, a washout period might be advisable when transitioning to alemtuzumab $[64,71]$. Unfortunately, for both fingolimod and DMF, the time period over which lymphocytes return to normal is variable and can take many weeks, during which time the patient is at risk of relapse [64].

Teriflunomide is also associated with a reduction ( $\sim 15 \%$, mean within normal limits) in lymphocytes and neutrophil counts within the first 3 months following treatment; mean lymphocyte and neutrophil counts then remain within the normal range for white blood cell counts $\left(3.8-10.7 \times 10^{9} / \mathrm{L}\right)$ during treatment [72]. Patients receiving teriflunomide have the opportunity to undergo an accelerated elimination procedure, which can reduce plasma levels by $>96 \%$ in 11 days [66, 73], potentially allowing the initiation of alemtuzumab relatively quickly after stopping teriflunomide.

By contrast, natalizumab does not reduce circulating lymphocyte counts, rather it blocks their entry to the CNS resulting in only mild lymphocytosis [64, 74], and there may be limited benefit in delaying initiating treatment with another DMT following natalizumab discontinuation [64]. In fact, it has been suggested that starting a new treatment immediately after stopping natalizumab (i.e., no a washout period) may be preferable because the risk of developing PML, even in anti-JCV antibody-positive patients, is lower than the risk of a severe relapse $[75,76]$. In CARE-MS II, patients previously treated with natalizumab (3\%) underwent a 6-month washout period before starting alemtuzumab [12]. In real-world clinical settings, and, in 
contrast with clinical studies, it is likely that a washout period of no more than 2 months following natalizumab discontinuation would be desirable owing to the risk of rebound disease $[64,76,77]$. Indeed, there are suggestions that use of a washout period when switching from natalizumab may do more harm than good [78].

In this regard, long-term real-world data would be of immense value in providing additional information on switching strategies between different DMTs. A recent real-world switching study demonstrated that in the 6 months post-natalizumab treatment, none of the 200 patients who switched to alemtuzumab experienced a relapse. In addition, 43\% (69/162) of patients with EDSS measurements showed improvements in their EDSS scores and only 1\% (2/162) had EDSS worsening. Of patients with MRI data, $2 \%(3 / 160)$ demonstrated new lesions on MRI. Generally, AEs were mild and easily managed with few serious AEs (one death occurred because of urinary tract infection and unrecognized non-convulsive status epilepticus) and no cases of PML were detected. The average washout period during this study was $\sim 9$ weeks [79]. Similar results were also observed in a second study from the same center, which evaluated outcomes in 250 patients who switched to alemtuzumab from prior DMTs, the majority $(66 \%)$ having previously received natalizumab [80].

Despite beneficial outcomes in the absence of a prolonged washout period, it is important when switching from natalizumab to alemtuzumab (or indeed to any other DMT) to confirm JCV status and exclude any PML carryover in JCV-positive patients by MRI and, in some cases, by cerebrospinal fluid examination for JCV DNA, before treatment commences. Although JCV status was established in the natalizumab switching studies discussed previously, it was not reported that PML was excluded prior to initiation of alemtuzumab [79, 80]. This is important, as the effects of alemtuzumab cannot be reversed in the short term, and, should carry-over PML develop following alemtuzumab treatment, it is unlikely that full immune cell repopulation will have occurred and patients will be unable to clear the virus. A bridging strategy may be useful in this case, where an alternative DMT or intravenous corticosteroids (methylprednisolone $1000 \mathrm{mg}$ ) can be used to prevent rebound disease, affording the opportunity to fully exclude PML [78, 81].

\subsection{Alemtuzumab in Highly Active Disease}

In a pre-specified subgroup analysis of the CARE-MS I and II studies, alemtuzumab demonstrated beneficial effects in patients with highly active disease (two or more relapses in the year prior to randomization and one or more $\mathrm{Gd}^{+}$lesion at baseline) who were treatment naïve [82] or had relapsed on prior DMTs [83]. In these highly active disease subgroups, alemtuzumab reduced AAR and time to 6-month CDW by a greater extent than SC IFN $\beta-1 \mathrm{a}$ and increased the number of years free from relapse. In addition, more patients were free of clinical and MRI disease activity [82, 83]. This is an important and clinically relevant observation, as this subgroup represents patients for whom robust, early intervention would be beneficial (treatmentnaive patients) or for whom switching therapies may be most appropriate (patients who had not responded to prior treatments).

\subsection{Levels of Disease Activity Required for Initiating Alemtuzumab}

Despite arguments against providing guidance on specific patient profiles, some clarification regarding the degree of disease activity required before considering alemtuzumab would be helpful. In general, clinical activity, or a combination of both clinical and MRI activity, should be present before treatment initiation (Table 6). However, it is important to consider each patient on a case-by-case basis and correlate any MRI findings with clinical activity. Relying solely on MRI findings alone could prove misleading, for example, in patients with pseudotumoral forms of MS, where single large lesions $(>2 \mathrm{~cm})$ mimic other tumor-like lesions such as neoplasms, infections, or infarctions [84, 85]. Guidance on how to effectively use

Table 6 Author recommendations for level of disease activity required for use of alemtuzumab

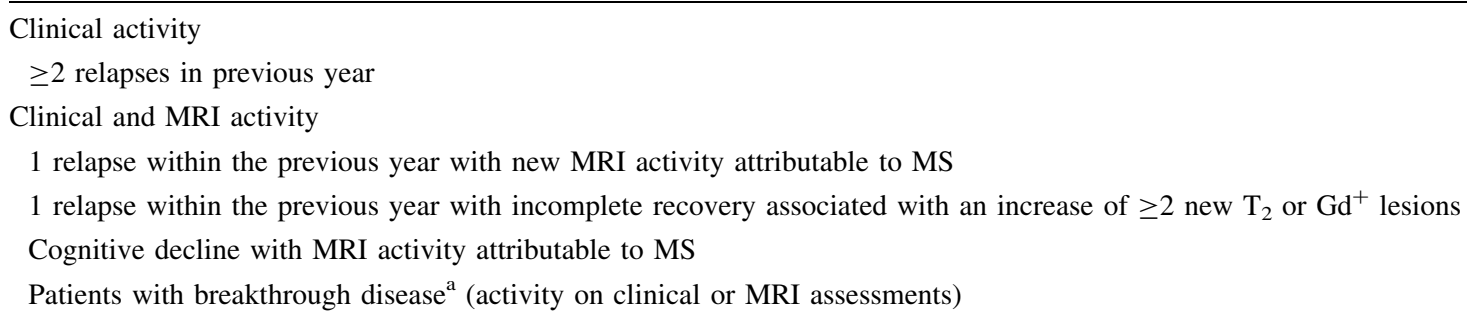

$G d^{+}$gadolinium-enhancing, MRI magnetic resonance imaging, $M S$ multiple sclerosis

${ }^{\text {a }}$ Patients with stable disease for several years who suddenly experience $\geq 1$ relapse with an increase in disability and number of $\mathrm{Gd}^{+}$lesions 
MRI findings to assist treatment decisions should also be provided to physicians.

The alemtuzumab EU label defines active disease as the presence of clinical or imaging features but gives no guidance as to the level of disease activity required before considering initiating therapy or retreatment [2]. In the three clinical studies [9, 11, 12], slightly differing criteria were used to define active disease, and MRI activity was only used to define active disease in CAMMS223 (Table 1) [9, 13]. Nevertheless, MRI activity was part of the retreatment criteria (see footnote to Table 1) applied in the CAMMS223 clinical trial (Sanofi Genzyme, data on file) and the CARE-MS extension studies [15].

\subsection{Patients Unsuitable for Alemtuzumab}

Currently, patients with inactive disease or those stable on current therapy (as per the approved label) would not be considered for treatment, and, as alemtuzumab has not been thoroughly evaluated in progressive MS, it is also not indicated for use in these patients [2]. Of course, there are always exceptions, and, while some patients may meet eligibility criteria as per the EU indication, certain safety considerations and challenges with frequent post-treatment monitoring may preclude them from being considered suitable for treatment. In such instances, a physician's experience and knowledge of their patient will prove invaluable in deciding upon the best approach. Compliance with the monitoring requirements associated with alemtuzumab treatment is vital to ensure identification of AEs promptly; therefore, a physician's previous experience will enable them to make an educated assessment with regard to their patients' suitability and likelihood of their adherence to the strict monitoring requirements. As alemtuzumab treatment is associated with a potential increased risk of developing serious infections, patients with signs of infectious disease may be precluded from initiating treatment until the infection is fully under control or has resolved [65]. Likewise, given that alemtuzumab may increase the chance of developing certain cancers (thyroid cancer, skin cancer [melanoma], lymphoproliferative disorders, and lymphoma) caution should be exercised before initiating treatment with alemtuzumab in patients with a pre-existing and/or an ongoing malignancy [2, 86].

\subsection{Expectations for Alemtuzumab Treatment}

Alemtuzumab is currently considered one of the most highly effective DMTs available for RRMS and provides patients with a unique treatment approach that results in durable efficacy in the absence of continuous treatment. Nevertheless, despite superior efficacy vs. subcutaneous IFN $\beta$-1a in treatment-naïve patients with active disease, alemtuzumab, with its long-term safety monitoring requirements is unlikely to replace the first-line DMTs such as IFN $\beta$, glatiramer acetate, teriflunomide, or DMF in the near term. Furthermore, given the complex nature of MS, with both inflammatory and neurodegenerative components, it remains to be determined whether alemtuzumab treatment can achieve permanent remission of MS disease activity over the very-long term. Extensions to the clinical trials are currently ongoing, and 4-year follow-up data have demonstrated that the majority of patients (74\% CARE-MS I; $66 \%$ CARE-MS II) have stable or improved disability at year 4 [7, 8], despite most (74\% CARE-MS I; 68\% CAREMS II) only receiving the initial two courses of alemtuzumab $[7,8]$.

\section{Effective Administration of Alemtuzumab}

In accordance with the EU label, alemtuzumab treatment should be supervised by a neurologist experienced in the treatment of patients with MS [2]. In addition, given the increased risk of autoimmune AEs during alemtuzumab treatment, it is encouraged that specialists who are able to provide treatment for autoimmune conditions be identified in advance of treatment initiation [2]. Although serious IARs only occurred in $\leq 3 \%$ of patients in the clinical trial setting [9, 11-13, 21, 23, 24], specialists and equipment required for the management of infusion-related conditions (e.g., hypersensitivity reactions) should also be available.

To minimize and reduce the severity of IARs, prophylactic antihistamines and antipyretics are recommended before alemtuzumab infusions [87]. Routine dosages of antihistamines, such as the non-sedating histamine $\mathrm{H}_{1}$-receptor antagonists (loratadine or cetirizine) and the $\mathrm{H}_{2}$ receptor antagonists (ranitidine or famotidine), may be considered, as well as antipyretics, such as acetaminophen or ibuprofen. These should be administered the morning of the alemtuzumab infusion, with a record being made of the time that the medication was taken [88].

In addition, intravenous corticosteroids may also be administered immediately prior to treatment on each of the first 3 days of any treatment course. However, in Italy, a recent study showed that around $50 \%$ of patients require intravenous corticosteroids (methylprednisolone $1000 \mathrm{mg}$ ) on days 4 and 5 of the first alemtuzumab infusion, as IARs were not reduced in severity with the standard 3 days of corticosteroid treatment [89]. This approach is now becoming more common in European clinics.

Oral prophylaxis with an anti-herpes agent (e.g., acyclovir $200 \mathrm{mg}$ twice daily) on the first day of each treatment course and continued for a minimum of 1 month is also recommended [2, 14]. 
Among other common AEs linked to IARs with alemtuzumab are an increased heart rate and a reduction in blood pressure; therefore, vital signs prior to, during, and for $2 \mathrm{~h}$ following the infusion period should be monitored [2].

Most alemtuzumab-related IARs can be managed with medical intervention or by slowing or interrupting the infusion [88]. Although the label recommends that alemtuzumab be administered as two annual courses given on 5 consecutive days at baseline and on 3 consecutive days 12 months later, respectively, with each daily infusion lasting $4 \mathrm{~h}$, dosing interruptions (e.g., a missed infusion) or an extension to the infusion period was permitted in clinical trials [2, 21, 90]. Infusion interruption in the CARE-MS studies was rare, with $95 \%$ of patients completing the initial course within 5-7 days and 97\% completing the second course within 3-5 days [21, 90]. In a recent study using data from CARE-MS I and II, administration of alemtuzumab infusions over an additional 5 days did not increase the overall incidence of IARs, affect the extent of lymphocyte depletion, or reduce treatment efficacy [90]. However, missed doses should not be given on the same day as a scheduled dose [2]. Patients may also receive additional courses of alemtuzumab beyond the first two annual courses, provided 12 months have passed since the previous course of treatment [2]. Criteria used in the CAMMS223 (Sanofi Genzyme, data on file) and the CARE-MS extensions [15] for retreatment may be used as a guide for when to initiate retreatment (Table 1).

The duration of alemtuzumab infusions may present some logistical issues, for example, where the patients will receive treatment. In Europe, this is likely to be in a specialist hospital-based setting; therefore, in countries with few MS centers, accessibility could impact access to treatment.

In clinical trials, most participants received alemtuzumab as outpatients, and this may prove more convenient in a real-world setting. For those patients who need to travel long distances to an MS center, hospitalization may be a more logical approach, and, in some cases, hospital admission may provide an opportunity for patients to receive additional services.

\section{Risk Minimization and Patient Monitoring}

\subsection{Vaccination Prior to Initiating Alemtuzumab Treatment}

The ability to mount effective immune responses to vaccines following alemtuzumab has not been studied extensively. However, a small pilot study demonstrated that alemtuzumab did not impair humoral immunologic memory with respect to the ability to generate antibodies against common viruses or the response to recall antigens, or the ability to mount a humoral immune response against a novel antigen [91]. The alemtuzumab label recommends that patients should complete local immunization requirements at least 6 weeks before starting therapy [2, 92]. Patients without a history of chicken pox should be tested and, if necessary, immunized against varicella zoster virus prior to starting treatment [2]. Immune responses against live viral vaccines have not been evaluated and current recommendations state that patients recently initiating alemtuzumab (as with all DMTs) should not receive live viral vaccines [2]; ongoing studies are evaluating the use of live viral vaccines in patients receiving alemtuzumab.

\subsection{Alemtuzumab Treatment-Related Risks}

In clinical studies, a number of risks were associated with alemtuzumab treatment, including IARs, infections, and autoimmune disorders, which led to establishment of an RMP in the EU (Table 7) and the REMS in the USA [30, 86]. Global labeling recommends that all patients receiving alemtuzumab need to be monitored for 48 months following their last alemtuzumab infusion [2, 3]. Data on identified (e.g., autoimmune disorders, including thyroid disorders, ITP, and glomerulonephropathy) and potential risks (e.g., malignancy and other autoimmune disorders, i.e., cytopenias) will be evaluated as per the RMP and REMS [30, 86].

Monitoring forms a significant and important component of alemtuzumab treatment and will be achieved through planned post-authorization safety studies, registries (including a pregnancy registry to establish any effects of alemtuzumab on pregnancy, lactation, and reproductive toxicity), and routine pharmacovigilance. As required by the RMP and REMS, the summary of product characteristics and educational materials (healthcare professional guide, prescriber checklist, patient guide, and alert card) have been made available to communicate the serious risks associated with the use of alemtuzumab [2]. These materials, in addition to counseling, hopefully will promote adherence to the monitoring program by highlighting the benefit-risk profile and reinforcing the importance of monitoring among both physicians and their patients.

Education and training of all medical staff (neurologists, general practitioners, and nurses) on the practicalities of administering alemtuzumab, particularly with regard to IARs, are key, so that patients get timely management if and when IARs occur. However, it is most important to ensure that patients are informed of the risks associated with alemtuzumab infusion, so that they can be reassured that IARs are an expected AE and the chance of 
Table 7 Risk management program and mitigation strategy [30, 86]

\begin{tabular}{|c|c|c|c|c|c|c|}
\hline & \multicolumn{6}{|c|}{ Mitigation strategy and planned actions } \\
\hline & $\begin{array}{l}\text { Prior to } \\
\text { treatment }\end{array}$ & Every month & $\begin{array}{l}\text { Every } 3 \\
\text { months }\end{array}$ & Education & $\begin{array}{l}\text { Routine } \\
\text { PV }\end{array}$ & PASS \\
\hline \multicolumn{7}{|l|}{ Identified risks } \\
\hline ITP $^{\mathrm{a}}$ & $x$ & $x$ & & $\times$ & $x$ & $x$ \\
\hline \multicolumn{7}{|l|}{ Test: CBC with differential } \\
\hline Nephropathies ${ }^{\mathrm{a}}$ & $\times$ & $\times$ & & $\times$ & $\times$ & $x$ \\
\hline \multicolumn{7}{|c|}{ Tests: serum creatinine levels, urinalysis with microscopy } \\
\hline Thyroid event $^{\mathrm{a}}$ & $\times$ & & $\times$ & $\times$ & $\times$ & $x$ \\
\hline Test: thyroid function (TSH levels) & & & & & & \\
\hline
\end{tabular}

ITP: generally responded to first-line treatment promptly

Nephropathies: no cases recorded in phase III studies (one membranous glomerulonephritis in the extension)

Thyroid events: managed with conventional medications/treatment; surgery was rare (one patient underwent splenectomy during CARE-MS II extension)

$C B C$ complete blood count, ITP immune thrombocytopenic purpura, PASS Post-Authorization Safety Study, PV pharmacovigilance, TSH thyroid-stimulating hormone

${ }^{a}$ Safety monitoring and education for early detection, proposed to continue for 48 months after last alemtuzumab infusion

infusion interruption can be minimized. Patients also need to be informed and understand the risks of serious autoimmune disorders, infections, and malignancies and the measures to minimize them, such as ongoing monitoring. A well-informed and educated patient will understand their responsibility to comply with the longterm monitoring requirements associated with alemtuzumab treatment. A plan, formulated between the physician and patient for monitoring post-alemtuzumab infusion is essential, and patients need to be made aware of the signs and symptoms of possible serious AEs. Consideration should be given to enrolling patients in programs such as the Sanofi Genzyme MS one-to-one program [93] or establishing a monthly e-mail reminder service to notify them when their monthly blood and urine tests are due. If potential logistical or social issues are known at the outset, physicians must consider whether these patients are suitable for alemtuzumab treatment. The greatest logistical problem, however, will be ensuring that those already receiving treatment adhere to the monitoring program, particularly those who respond well after two courses of treatment and have no evidence of active disease.

As part of the RMP and REMS, mechanisms will be in place to remind patients about their monthly monitoring appointments and also to ensure that physicians are informed if tests are missed [30, 86]. It may be pertinent to implement an active follow-up service, perhaps coordinated through the patient's general practitioner, to ensure they are adhering to the monitoring requirements. This may be particularly important for patients who do not live in close proximity to a MS center. These approaches will help facilitate early detection of AEs, increasing the likelihood of favorable outcomes. The RMP and REMS are dynamic and will be updated if new information emerges leading to a significant change in the benefit-risk profile or if an important pharmacovigilance or risk minimization milestone is reached [2].

\subsection{Autoimmune Adverse Events}

The RMP and REMS have been designed to ensure early detection of autoimmune conditions, such as thyroid disorders and, more rarely, ITP, glomerulonephropathies, and other autoimmune disorders. Testing should begin in all patients prior to initiation of therapy and continue on a monthly basis for 48 months following the last infusion $[2,30]$.

Thyroid dysfunction is the most commonly reported autoimmune $\mathrm{AE}$ in alemtuzumab-treated patients, with the thyroid gland being adversely affected in $\sim 36 \%$ of patients in a 4-year follow-up of the CARE-MS I and II trials [94]. Autoimmune thyroid disease may lead to hyperthyroidism (increased thyroid hormone levels in the serum with suppressed thyroid-stimulating hormone) or hypothyroidism and neurologists need to be aware of how to diagnose and treat these conditions [94]. Typically, changes in thyroid-stimulating hormone, thyroxine, and triiodothyronine levels are evaluated $[95,96]$. In some centers, thyroid antibodies are also investigated and where hyper- or hypothyroidism is suspected these cases are followed up with an ultrasound examination of the thyroid gland. A recent article by Mahzari et al. presents case studies for four patients from the CARE-MS trials who 
developed autoimmune thyroid disease and provides a comprehensive account of the signs and symptoms to be aware of [94].

Alemtuzumab-induced thyroid disease is a treatmentrelated $\mathrm{AE}$ that needs to be recognized, as generally it can be managed relatively easily with standard-of-care treatments. Management involves differentiating the underlying etiology, e.g., establishing whether this is due to hypothyroidism or hyperthyroidism and whether Graves' disease or subacute painless thyroiditis is the cause of hyperthyroidism. In the case of hypothyroidism, thyroid hormone replacement therapy should be considered, with patients monitored every 4-8 weeks to adjust thyroid hormone dosages. If hypothyroidism develops following treatment for hyperthyroidism, subacute painless thyroiditis should be suspected. Hyperthyroidism following alemtuzumab treatment is most likely due to Graves' disease and should be managed initially with anti-thyroid medication (thionamides); in clinical trials, this approach, rather than radioactive iodine or thyroidectomy, was associated with a higher likelihood of remission. Thyroidectomy or radioactive iodine would be indicated following failure of anti-thyroid medication. Where subacute painless thyroiditis is suspected, $\beta$-adrenergic blockers or corticosteroids (severe cases only) may be considered. Anti-thyroid medications are not applicable in these patients as thyroid hormone synthesis is already low. An algorithm for the treatment of patients presenting with alemtuzumab-induced hyperthyroidism has been proposed by Mahzari et al. [94].

Thyroid disease poses a special risk in women who are pregnant, and studies are currently underway to investigate the effect of alemtuzumab on fertility and pregnancy $[2,97]$. Patients should also be instructed on how to recognize the signs and symptoms of ITP, such as appearance of small spots on the skin (petechiae), easy bruising, heavy menstruation, bleeding that takes longer than normal to stop, or hematoma development, and informed about other risk factors for autoimmune disease (e.g., smoking and a family history of autoimmune disease) [98, 99]. If signs of ITP are observed, patients should have a complete blood count to determine whether their platelet number has reduced, and, if ITP is confirmed, the patient should be immediately referred to a specialist.

In the core clinical trials, ITP was reported in 16 patients (CAMMS223 [ $n=6$ ]; CARE-MS I [ $n=3$ ] and CARE-MS II $[n=7])$ and occurred between 1 and 16 months after the last alemtuzumab infusion. With the exception of the fatal index case (cerebral hemorrhage), most cases resolved spontaneously $(n=2)$, or after treatment with platelet transfusion, rituximab, steroids and/or intravenous immunoglobulin, and within 3 months of onset. One patient from the CARE-MS II study underwent splenectomy following corticosteroid treatment [12]. In the extension study, a total of 20 cases of ITP were identified from patients originating from the CAMMS223 $(n=1)$, CARE-MS I $(n=4)$, and CARE-MS II $(n=15)$ studies. In these patients, onset occurred between 4 and 44 months after the last alemtuzumab infusion and, as with the core studies, resolved spontaneously $(n=1)$ or following standard treatment(s) within 3.2 months (Sanofi Genzyme, data on file).

More extensive details with regard to ITP cases occurring in the alemtuzumab clinical trial program will be published elsewhere. All treatment-emergent ITP events observed in the CARE-MS core and extension studies were identified through the routine monitoring of platelets and/or patient reporting of signs or symptoms of ITP established following the fatal index case in CAMMS223, highlighting the effectiveness of the monitoring program and educational materials used routinely in these studies (Sanofi Genzyme, data on file) [29]. The RMP in the EU and REMS in the USA are based on these monitoring programs and educational materials.

Immune nephropathies (including anti-GBM disease) were detected in $0.3 \%$ of patients throughout the clinical trials and extensions [9, 11-13, 15]. Anti-GBM disease can result in severe kidney damage, thus urine should be monitored closely [14]. Any detection of clinically significant changes from baseline in serum creatinine, hematuria, proteinuria, and urine cell counts (blood and epithelial cells) and the presence of antiGBM antibodies should prompt immediate referral to a nephrologist, as it is often difficult to determine the etiology of abnormalities given that urinary tract infections and hematuria are common occurrences in patients with MS [31, 99].

Therefore, it is important to ensure that, when establishing an alemtuzumab service, sufficient infrastructure is in place that can not only deliver treatment effectively but also can detect any AEs and manage them effectively via a predefined network of specialists familiar with alemtuzumab and its autoimmune effects.

\subsection{Malignancies}

Alemtuzumab has a long-term effect on the immune system, and, as with other immunomodulatory therapies, the potential for malignancies is an area of concern. Therefore, caution is recommended when using alemtuzumab in patients with a pre-existing and/or ongoing malignancy [2]. Annual malignancy rates during the controlled phases of the clinical trials were the same for alemtuzumab and SC IFN $\beta$-1a (0.003 events/year), and the risk of all cancers combined was significantly lower than in the reference population [100]. The European Medicines Agency also 
concluded that rates of malignancy are no different from the general population, although firm conclusions regarding the risk could not be drawn [92].

\section{Conclusion}

This article has discussed the various factors that physicians and patients in Europe will need to consider when initiating alemtuzumab treatment. One of the main questions for physicians considering alemtuzumab will be to determine which patients are most suitable. The EU label for alemtuzumab is broad, and defining a specific patient profile for alemtuzumab may not be necessary, or even possible. In fact, it may be more useful to determine which patients are unsuitable for treatment, such as those with inactive disease or who are stable on current therapy in accordance with the label, or for those who may be unable to comply with the monitoring requirements.

Suitable patients fall into two groups, active treatmentnaïve patients and patients only having a partial response to their current DMT. Alemtuzumab may therefore find use as an initial therapeutic in treatment-naive patients with active MS, although the use of a drug with the benefit-risk profile of alemtuzumab early in the disease course may require a change in the way physicians balance the risks of disease worsening against those of treatment. The efficacy of alemtuzumab in patients with highly active disease suggests that it could also be used as escalation therapy in patients not fully responding to their current DMTs or in patients needing to switch therapies for other reasons (e.g., safety/tolerability, poor adherence).

Clearly, alemtuzumab has an important place in the MS treatment paradigm, but it is unlikely to replace first-line DMTs in the short term. While there are logistical challenges associated with patient monitoring, these could be offset by the absence of daily, weekly, or monthly dosing regimens along with initial and durable efficacy benefits.

Given the complex nature of MS, with both inflammatory and neurodegenerative components, it remains to be seen whether alemtuzumab treatment can achieve a permanent remission of MS disease activity. Nevertheless, alemtuzumab represents a unique treatment option for patients with RRMS, providing durable efficacy in the absence of continuous treatment and, thus, offering physicians and patients a wider treatment choice for controlling MS.

Acknowledgements This manuscript was reviewed by Darren P. Baker PhD, and Neli Boyanova MD, of Sanofi Genzyme. Editorial support (assistance in drafting and editing of the manuscript text and tables, as directed by authors, data checking and incorporation of comments from reviewers, and assisting with the submission process) was provided by Steve Banner at Fishawack Communications Ltd., funded by Sanofi Genzyme. This was the only funding provided for the development of this manuscript. This article is based on the outcomes of a European Advisory Board, held in Vienna, Austria, in November 2013, during which a panel of European MS experts provided insights, guidance, and recommendations regarding best practices for alemtuzumab treatment and compliance with ongoing monitoring requirements designed to mitigate potential adverse events. Open access funding provided by University of Innsbruck and Medical University of Innsbruck.

\section{Compliance with Ethical Standards}

Funding The advisory board was funded by Sanofi Genzyme. All advisors received honoraria payments for their participation. Open access fee was funded by the Medical University of Innsbruck.

Conflict of interest Thomas Berger has received consulting fees or honorarium and payment for lectures including service on speaker's bureaus from Allergen, Bayer, Biogen, Genzyme, Merck, Novartis, Sanofi, and Teva, and support for travel from Genzyme. Irina Elovaara has received honoraria for participation in advisory boards and travel support from Genzyme. Sten Fredrikson has received honoraria for lectures, educational activities, or consultancy from Allergan, Bayer, Biogen, Genzyme, Merck, Novartis, Sanofi, and Teva. Chris McGuigan (and/or department) has received research grants from Biogen, Genzyme, Novartis, and Bayer, honoraria as a consultant for Biogen, Genzyme, and Novartis, and travel support from Genzyme. Lucia Moiola has received honoraria for speaking in a scientific meeting or advisory board from Biogen, Sanofi-Genzyme, Teva, and Novartis. Kjell-Morten Myhr has received honoraria for lecturing and participation in advisory boards or pharmaceutical company-sponsored clinical trials, and travel support from Allergan, Almirall, Bayer Schering, Biogen, Sanofi-Genzyme, Merck-Serono, Novartis, and Teva. Celia Oreja-Guevara has received honoraria from Biogen, Sanofi-Genzyme, Bayer, Novartis, Teva, and Merck-Serono. Igor Stoliarov has no conflicts of interest. Uwe Zettl has received speaker honoraria, travel support, and research support from Almirall, Bayer, Biogen, Genzyme, Merck Serono, Novartis, and Sanofi Aventis.

Open Access This article is distributed under the terms of the Creative Commons Attribution-NonCommercial 4.0 International License (http://creativecommons.org/licenses/by-nc/4.0/), which permits any noncommercial use, distribution, and reproduction in any medium, provided you give appropriate credit to the original author(s) and the source, provide a link to the Creative Commons license, and indicate if changes were made.

\section{References}

1. Dayan C, Cuker A, LaGanke C, et al. Autoimmunity in patients treated with alemtuzumab for relapsing-remitting multiple sclerosis: 5-year follow-up of the CARE-MS studies. ECTRIMS meeting. 14-17 Sept 2016; London, UK: platform 168.

2. EMA. Lemtrada (alemtuzumab): EU summary of product characteristics. 2014. Available from: http://www.ema.europa.eu/ docs/en_GB/document_library/EPAR_-_Product_Information/ human/003718/WC500150521.pdf. Accessed Oct 2015.

3. US FDA. Lemtrada: prescribing information. 2016. Available from: http://www.accessdata.fda.gov/drugsatfda_docs/label/ 2014/103948s5139lbl.pdf. Accessed Oct 2015.

4. Hu Y, Turner MJ, Shields J, et al. Investigation of the mechanism of action of alemtuzumab in a human CD52 transgenic mouse model. Immunology. 2009;128(2):260-70. 
5. Turner MJ, Lamorte MJ, Chretien N, et al. Immune status following alemtuzumab treatment in human CD52 transgenic mice. J Neuroimmunol. 2013;261(1-2):29-36.

6. Klotz L, Meuth SG, Wiendl H. Immune mechanisms of new therapeutic strategies in multiple sclerosis: a focus on alemtuzumab. Clin Immunol. 2012;142(1):25-30.

7. Havrdova E, Giovannoni G, Arnold D, et al. Durable effect of alemtuzumab on clinical outcomes in patients with relapsingremitting multiple sclerosis who relapsed on prior therapy: 4-year follow-up of CARE-MS II. In: 67th annual meeting of the American Academy of Neurology; 18-25 Apr 2015; Washington, DC: P7.276.

8. Compston A, Giovannoni G, Arnold D, et al. Durable effect of alemtuzumab on clinical outcomes in treatment-naïve relapsingremitting multiple sclerosis patients: 4-year follow-up of CAREMS I. In: 67th annual meeting of the American Academy of Neurology; 18-25 Apr 2015; Washington, DC: S4.007.

9. Coles AJ, Compston DA, Selmaj KW, et al. Alemtuzumab vs. interferon beta-1a in early multiple sclerosis. N Engl J Med. 2008;359(17):1786-801.

10. ClinicalTrials.gov. An extension protocol for multiple sclerosis patients who participated in Genzyme-sponsored studies of alemtuzumab. 2009. Available from: https://www.clinicaltrials. gov/ct2/show/NCT00930553. Accessed Oct 2015.

11. Cohen JA, Coles AJ, Arnold DL, et al. Alemtuzumab versus interferon beta 1a as first-line treatment for patients with relapsing-remitting multiple sclerosis: a randomised controlled phase 3 trial. Lancet. 2012;380(9856):1819-28.

12. Coles AJ, Twyman CL, Arnold DL, et al. Alemtuzumab for patients with relapsing multiple sclerosis after disease-modifying therapy: a randomised controlled phase 3 trial. Lancet. 2012;380(9856):1829-39.

13. Coles AJ, Fox E, Vladic A, et al. Alemtuzumab more effective than interferon beta-1a at 5-year follow-up of CAMMS223 clinical trial. Neurology. 2012;78(14):1069-78.

14. Sanofi Genzyme Corporation. Lemtrada in depth: managing disease progression in patients with relapsing remitting multiple sclerosis. 2013; Cambridge, Sanofi Genzyme.

15. Fox EJ, Arnold DL, Cohen JA, et al. Durable efficacy of alemtuzumab in relapsing-remitting multiple sclerosis patients who participated in the CARE-MS studies: three year follow-up. In: 65th annual meeting of the American Academy of Neurology; 16-23 Mar 2013; San Diego (CA): S41.001.

16. Fox EJ, Wynn D, Coles AJ, Palmer J, Margolin DH, Investigators C. Alemtuzumab improves neurological functional systems in treatment-naïve relapsing-remitting multiple sclerosis patients. J Neurol Sci. 2016;363:188-94.

17. Coles A, Arnold D, Cohen J, et al. Alemtuzumab slows brain volume loss over 4 years despite most relapsing-remitting multiple sclerosis patients not receiving treatment for 3 years. In: 67th annual meeting of the American Academy of Neurology; 18-25 Apr 2015; Washington, DC: P7.263.

18. Arnold D, Traboulsee A, Coles A, et al. Durable effect of alemtuzumab on MRI activity in treatment-naïve active relapsing-remitting multiple sclerosis patients: 4-year followup of CARE-MS I. In: 67th annual meeting of the American Academy of Neurology; 18-25 Apr 2015; Washington, DC: P7.246.

19. Traboulsee A, Coles A, Cohen J, et al. Durable effect of alemtuzumab on MRI outcomes in patients with relapsingremitting multiple sclerosis who relapsed on prior therapy: 4-year follow-up of CARE-MS II. In: 67th annual meeting of the American Academy of Neurology; 18-25 Apr 2015; Washington, DC: 2015: P7.249.

20. Boyko AN, on behalf of the CaMMS223 Study Group. Alemtuzumab's durable efficacy in multiple sclerosis four years after last treatment cycle. ECTRIMS Meeting. 19-22 Oct 2011; Amsterdam, the Netherlands; P931.

21. Mayer L, Casady L, Clayton G, et al. Management of infusionassociated reactions in alemtuzumab-treated relapsing-remitting multiple sclerosis patients. ACTRIMS-ECTRIMS Meeting; 10-13 Sept 2014; Boston, MA, USA; P880.

22. Caon C, Namey M, Meyer C, et al. Prevention and management of infusion-associated reactions in the comparison of alemtuzumab and rebif $\left({ }^{\circledR}\right)$ efficacy in multiple sclerosis (CARE-MS) program. Int J MS Care. 2015;17(4):191-8.

23. LaGanke C, Arnold D, Cohen J, et al. Adverse event profile of alemtuzumab over time in active relapsing-remitting multiple sclerosis patients who experienced disease activity while on prior therapy (CARE-MS II). Neurology. 2013;80(Meeting Abstracts 1):P01.174.

24. Lycke J, Arnold D, Cohen J, et al. Adverse event profile of alemtuzumab over time in treatment-naïve patients with early, active relapsing-remitting multiple sclerosis (CARE-MS I study). 21st World Congress of Neurology; 21-26 Sep 2013; Vienna: P202.

25. Havrdova E, Arnold D, Hartung H, et al. Infection risk with alemtuzumab in patients with relapsing-remitting multiple sclerosis: pooled results from the CARE-MS I and II trials. ECTRIMS Meeting; 2-5 Oct 2013; Copehagen, Denmark; P603.

26. Twyman C, Oyuela P, Palmer J, et al. Thyroid autoimmune adverse events in patients treated with alemtuzumab for relapsing-remitting multiple sclerosis: four-year follow-up of the CARE-MS studies. In: 66th annual meeting of the American Academy of Neurology; Apr 26-May 3 2014; Philadelphia (PA); 82(10 Suppl.):P2.199.

27. Miller T, Arnold D, Cohen J, et al. Detection, incidence, and management of thyroid autoimmunity in comparison of alemtuzumab and rebif ${ }^{\circledR}$ in multiple sclerosis (CARE-MS) I and II. In: 65th annual meeting of the American Academy of Neurology; 16-23 Mar 2013; San Diego (CA): P01.173.

28. Cuker A, Coles AJ, Sullivan H, et al. A distinctive form of immune thrombocytopenia in a phase 2 study of alemtuzumab for the treatment of relapsing-remitting multiple sclerosis. Blood. 2011;118(24):6299-305.

29. Cuker A, Stasi R, Palmer J, et al. Successful detection and management of immune thrombocytopenia in alemtuzumabtreated patients with active relapsing-remitting multiple sclerosis. Neurology. 2014;82(10 Suppl):P2.198.

30. US FDA. Lemtrada (alemtuzumab): risk evaluation and mitigation strategy (REMS). 2014:1-82. http://www.fda.gov. Accessed Nov 2014.

31. Wynn DR, Arnold D, Cohen J, et al. Detection, incidence and management of glomerulonephritis in the alemtuzumab clinical development programme. ECTRIMS Meeting; 2-5 Oct 2013; Copehagen, Denmark; P597.

32. Hartung H-P, Aktas O, Boyko AN. Alemtuzumab: a new therapy for active relapsing-remitting multiple sclerosis. Mult Scler. 2015;21(1):22-34.

33. Coles A, Arnold D, Cohen J, et al. Efficacy and safety of alemtuzumab in treatment-naïve patients with relapsing-remitting MS: four-year follow-up of the CARE-MS I study. ACTRIMS-ECTRIMS Meeting; 10-13 Sept 2014; Boston, MA, USA; P090.

34. Arnold D, Brinar V, Cohen J, et al. Effect of alemtuzumab vs. Rebif $^{\circledR}$ on brain MRI measurements: results of CARE-MS I, a phase 3 study. In: 64th annual meeting of the American Academy of Neurology; 21-24 Apr 2012; New Orleans (LO): S11.006.

35. Arnold D, Cohen J, Coles A, et al. Effect of alemtuzumab vs. Rebif $^{\circledR}$ on brain MRI measurements: results of CARE-MS II. ECTRIMS Meeting; 10-13 Oct 2012; Lyon, France; P877. 
36. Margolin D, Kieseier B, Palmer J, Ziemssen T. Alemtuzumab has similar efficacy and safety in active relapsing-remitting multiple sclerosis (RRMS) patients who were treatment-naïve or who relapsed on prior therapy. Neurology. 2014;82(10 Suppl):P2.209.

37. Tuohy O, Costelloe L, Hill-Cawthorne G, et al. Alemtuzumab treatment of multiple sclerosis: long-term safety and efficacy. J Neurol Neurosurg Psychiatry. 2014;86(2):208-15.

38. Hirst CL, Pace A, Pickersgill TP, et al. Campath 1-H treatment in patients with aggressive relapsing remitting multiple sclerosis. J Neurol. 2008;255(2):231-8.

39. EMA. Gilenya (fingolimod): EU summary of product characteristics. 2015. Available from: http://www.ema.europa.eu/docs/en GB/document_library/EPAR_-_Product_Information/human/002 202/WC500104528.pdf. Accessed Oct 2015.

40. EMA. Tysabri (natalizumab): EU summary of product characteristics. 2015. Available from: http://www.ema.europa.eu/docs/ en_GB/document_library/EPAR_-_Product_Information/human/ 000603/WC500044686.pdf. Accessed Oct 2015.

41. Twyman C, Montalban X, Arnold D, et al. Relapse outcomes with alemtuzumab vs IFN $\beta-1 \mathrm{a}$ in active relapsing-remitting multiple sclerosis patients who experienced disease activity while on prior therapy (CARE-MS II). In: 65th annual meeting of the American Academy of Neurology; 16-23 Mar 2013; San Diego (CA): P07.098.

42. Fox E, Arnold D, Brinar V, et al. Relapse outcomes with alemtuzumab vs. Rebif ${ }^{\circledR}$ in treatment-naïve relapsing-remitting multiple sclerosis (CARE-MS I): secondary and tertiary endpoints (PD5.004). In: 64th annual meeting of the American Academy of Neurology; 21-24 Apr 2012; New Orleans (LO): PD5.004.

43. Coles AJ, Fox E, Vladic A, et al. Alemtuzumab versus interferon beta-1a in early relapsing-remitting multiple sclerosis: post-hoc and subset analyses of clinical efficacy outcomes. Lancet Neurol. 2011;10(4):338-48.

44. Cossburn MD, Harding K, Ingram G, et al. Clinical relevance of differential lymphocyte recovery after alemtuzumab therapy for multiple sclerosis. Neurology. 2013;80(1):55-61.

45. Kousin-Ezewu O, Azzopardi L, Parker RA, et al. Accelerated lymphocyte recovery after alemtuzumab does not predict multiple sclerosis activity. Neurology. 2014;82(24):2158-64.

46. Robertson NP, Scolding NJ. Immune reconstitution and treatment response in multiple sclerosis following alemtuzumab. Neurology. 2014;82(24):2150-1.

47. Bermel RA, Bakshi R. The measurement and clinical relevance of brain atrophy in multiple sclerosis. Lancet Neurol. 2006;5(2):158-70.

48. Vollmer T, Huynh L, Kelley C, et al. Relationship between brain volume loss and cognitive outcomes among patients with multiple sclerosis: a systematic literature review. Neurol Sci. 2016;37(2):165-79.

49. Goodin DS, Reder AT, Ebers GC, et al. Survival in MS: a randomized cohort study 21 years after the start of the pivotal IFN $\beta-1 b$ trial. Neurology. 2012;78(17):1315-22.

50. Miller JR. The importance of early diagnosis of multiple sclerosis. J Manag Care Pharm. 2004;10(3 Suppl B):S4-11.

51. Noyes K, Weinstock-Guttman B. Impact of diagnosis and early treatment on the course of multiple sclerosis. Am J Manag Care. 2013;19(17 Suppl):s321-31.

52. Trapp BD, Peterson J, Ransohoff RM, et al. Axonal transection in the lesions of multiple sclerosis. $\mathrm{N}$ Engl $\mathrm{J}$ Med. 1998;338(5):278-85.

53. Wingerchuk DM, Carter JL. Multiple sclerosis: current and emerging disease-modifying therapies and treatment strategies. Mayo Clin Proc. 2014;89(2):225-40.

54. Hutchinson M, Kappos L, Calabresi PA, et al. The efficacy of natalizumab in patients with relapsing multiple sclerosis: subgroup analyses of AFFIRM and SENTINEL. J Neurol. 2009;256(3):405-15.

55. Bergamaschi R. Prognostic factors in multiple sclerosis. Int Rev Neurobiol. 2007;79:423-47.

56. Langer-Gould A, Popat RA, Huang SM, et al. Clinical and demographic predictors of long-term disability in patients with relapsing-remitting multiple sclerosis: a systematic review. Arch Neurol. 2006;63(12):1686-91.

57. Schwid SR, Bever CT Jr. The cost of delaying treatment in multiple sclerosis: what is lost is not regained. Neurology. 2001;56(12):1620.

58. PRISMS Study Group. University of British Columbia MS/MRI Analysis Group. PRISMS-4: long-term efficacy of interferonbeta-1a in relapsing MS. Neurology. 2001;56(12):1628-36.

59. Uitdehaag B, Constantinescu C, Cornelisse P, et al. Impact of exposure to interferon beta-1a on outcomes in patients with relapsing-remitting multiple sclerosis: exploratory analyses from the PRISMS long-term follow-up study. Ther Adv Neurol Disord. 2011;4(1):3-14.

60. Kappos L, Traboulsee A, Constantinescu C, et al. Long-term subcutaneous interferon beta-1a therapy in patients with relapsing-remitting MS. Neurology. 2006;67(6):944-53.

61. Kappos L, Freedman MS, Polman CH, et al. Long-term effect of early treatment with interferon beta- $1 \mathrm{~b}$ after a first clinical event suggestive of multiple sclerosis: 5-year active treatment extension of the phase 3 BENEFIT trial. Lancet Neurol. 2009;8(11):987-97.

62. Havrdova E, Horakova D, Kovarova I. Alemtuzumab in the treatment of multiple sclerosis: key clinical trial results and considerations for use. Ther Adv Neurol Disord. 2015;8(1): 31-45.

63. Bloomgren G, Richman S, Hotermans C, et al. Risk of natalizumab-associated progressive multifocal leukoencephalopathy. N Engl J Med. 2012;366(20):1870-80.

64. Miller AE. Switching or discontinuing disease-modifying therapies for multiple sclerosis. Continuum (Minneap Minn). 2016;22(3):851-63.

65. EMA. Aubagio (teriflunomide): EU summary of product characteristics. Available from: http://www.ema.europa.eu/docs/en GB/document_library/EPAR_-_Product_Information/human/002 514/WC500148682.pdf. 2015. Accessed 28 Oct 2016.

66. Kieseier BC, Benamor M. Pregnancy outcomes following maternal and paternal exposure to teriflunomide during treatment for relapsing-remitting multiple sclerosis. Neurol Ther. 2014;3(2):133-8.

67. EMA. Tecfidera (dimethyl fumarate): EU summary of product characteristics. Available from: http://www.ema.europa.eu/docs/ en_GB/document_library/EPAR_-_Product_Information/human/ 002601/WC500162069.pdf. 2014. Accessed 28 Oct 2016.

68. German-Austrian-Swiss MS Competence Network. MS treatment guidelines. http://www.kompetenznetz-multiplesklerose. de. Accessed Sep 2016.

69. Kappos L, Radue EW, O'Connor P, et al. A placebo-controlled trial of oral fingolimod in relapsing multiple sclerosis. N Engl J Med. 2010;362(5):387-401.

70. Willis M, Pearson O, Nielsen C, Sejbaek T, et al. Multiple sclerosis disease rebound after treatment switch from fingolimod to alemtuzumab. ECTRIMS Meeting; 14-17 Sept 2016; London, UK; P1258.

71. Fox RJ, Kita M, Cohan SL, et al. BG-12 (dimethyl fumarate): a review of mechanism of action, efficacy, and safety. Curr Med Res Opin. 2014;30(2):251-62.

72. Leist TP, Freedman MS, Kappos L, et al. Pooled safety analyses from teriflunomide clinical studies. Neurology. 2015;84 (Suppl.14):1526-632X:P7.268. 
73. Lunven $\mathrm{C}$, Guo Z, Turpault $\mathrm{S}$, et al. Investigation of the effectiveness and tolerability of colesevelam $\mathrm{HCl}$ for accelerated elimination of teriflunomide in healthy subjects. Annual meeting of the Consortium of Multiple Sclerosis Centers; 27-30 May 2015; Indianapolis, Indiana, USA; DX50.

74. Polman $\mathrm{CH}$, O'Connor PW, Havrdova E, et al. A randomized, placebo-controlled trial of natalizumab for relapsing multiple sclerosis. N Engl J Med. 2006;354(9):899-910.

75. Gallo P, Van Wijmeersch B. Overview of the management of relapsing-remitting multiple sclerosis and practical recommendations. Eur J Neurol. 2015;22(Suppl. 2):14-21.

76. Clerico M, Schiavetti I, De Mercanti SF, et al. Treatment of relapsing-remitting multiple sclerosis after 24 doses of natalizumab: evidence from an Italian spontaneous, prospective, and observational study (the TY-STOP Study). JAMA Neurol. 2014;71(8):954-60.

77. Sorensen PS, Koch-Henriksen N, Petersen T, et al. Recurrence or rebound of clinical relapses after discontinuation of natalizumab therapy in highly active MS patients. J Neurol. 2014;261(6):1170-7.

78. Frohman TC, Beh SC, Kildebeck EJ, et al. Neurotherapeutic strategies for multiple sclerosis. Neurol Clin. 2016;34(3):483-523.

79. LaGanke C, Adcock A. Clinical outcomes of 200 multiple sclerosis patients switching from natalizumab to alemtuzumab in a single United States MS center. In: 68th Annual meeting of the American Academy of Neurology; 15-21 Apr 2016; Vancouver (BC): P3.117.

80. LaGanke C, Adcock A. Early experience of 250 multiple sclerosis pateints treated with alemtuzumab in a single United States MS cCenter. In: 68th Annual Meeting of the American Academy of Neurology; 15-21 Apr 2016; Vancouver (BC): P3.086.

81. BartsMS Blog. Clinic Speak: switching from natalizumab to alemtuzumab. 2015. Available from: http://multiple-sclerosisresearch.blogspot.com/2014/05/clinic-speak-switching-fromnatalizumab.html. Accessed 28 Oct 2013.

82. Krieger S, Lubetzki C, Arnold D, et al. Alemtuzumab reduces disease activity in treatment-naïve patients with highly active relapsing-remitting multiple sclerosis. ACTRIMS-ECTRIMS Meeting; 10-13 Sept 2014; Boston, MA, USA; P088.

83. Krieger S, Arnold D, Cohen J, et al. Alemtuzumab is efficacious in highly-active RRMS patients in CARE-MS II. CMSCACTRIMS; 29 May-1 June 2013; Orlando, FL, USA; DX10.

84. Mauri-Fabrega L, Diaz-Sanchez M, Casado-Chocan JL, UclesSanchez AJ. Pseudotumoral forms of multiple sclerosis: report of 14 cases and review of the literature. Eur Neurol. 2014;72(1-2):72-8.

85. de Medeiros FC, de Albuquerque LA, Pittella JE, et al. Open-ring enhancement in pseudotumoral multiple sclerosis: important radiological aspect. Case Rep Neurol Med. 2014;2014:951690.
86. Sanofi Genzyme. Lemtrada (alemtuzumab): REMS (Risk Evaluation and Mitigation Strategy) program. 2016. Available from: https://www.lemtradarems.com/. Accessed 3 Aug 2016.

87. Lenz HJ. Management and preparedness for infusion and hypersensitivity reactions. Oncologist. 2007;12(5):601-9.

88. McEwan L, Caon C, Chieffe C, et al. Best practices in alemtuzumab administration: practical recommendations for infusion in patients with multiple sclerosis. J Infusion Nursing. 2016;39(2):93-104.

89. Annovazzi P, Prosperini L, Boffa M, et al. Alemtuzumab infusions under a magnifyng lens: data from the 40 first-treated Italian patients on timing and tolerability. XLVI Congress of Italian Neurological Society; 10-13 Oct 2015; Genova, Italy.

90. Wray S, Boyko AN, Braley T, et al. Administration of alemtuzumab on nonconsecutive days does not impact infusion-associated reactions, efficacy, or lymphocyte depletion. In: 67th annual meeting of the American Academy of Neurology; 18-25 Apr 2015; Washington, DC: P7.277.

91. McCarthy CL, Tuohy O, Compston DA, et al. Immune competence after alemtuzumab treatment of multiple sclerosis. Neurology. 2013;81(10):872-6.

92. EMA. Lemtrada assessment report. 2013. Available from: http:// www.ema.europa.eu/docs/en_GB/document_library/EPAR_-Public_assessment_report/human/003718/WC500150522.pdf. Accessed 28 Oct 2015.

93. Genzyme S. MS one to one. 2016. Available from: http:// msonetoone.eu/. Accessed 4 Aug 2016.

94. Mahzari M, Arnaout A, Freedman MS. Alemtuzumab induced thyroid disease in multiple sclerosis: a review and approach to management. Can J Neurol Sci. 2015;42(5):284-91.

95. British Thyroid Foundation. Quick guides: hyperthyroidism. 2016. Available from: http://www.btf-thyroid.org/information/ quick-guides/. Accessed 28 Oct 2016.

96. British Thyroid Foundation. Quick guides: hypothyroidism. 2016. Available from: http://www.btf-thyroid.org/information/ quick-guides/. Accessed 28 Oct 2016.

97. McCombe P, Achiron A, Giovannoni G, et al. Pregnancy outcomes in the alemtuzumab multiple sclerosis clinical development program. ACTRIMS-ECTRIMS Meeting; 10-13 Sept 2014; Boston, MA, USA; P842.

98. Cossburn M, Pace AA, Jones J, et al. Autoimmune disease after alemtuzumab treatment for multiple sclerosis in a multicenter cohort. Neurology. 2011;77(6):573-9.

99. Subei AM, Ontaneda D. Risk mitigation strategies for adverse reactions associated with the disease-modifying drugs in multiple sclerosis. CNS Drugs. 2015;29(9):759-71.

100. Miller T, Habek M, Coles A, et al. Analysis of data from RRMS alemtuzumab-treated patients in the clinical program to evaluate incidence rates of malignancy. ACTRIMS-ECTRIMS Meeting; 10-13 Sept 2014; Boston, MA, USA; P868. 